\section{$\underset{\substack{\text { hommes } \\ \text { \& migrations }}}{ }$}

\section{Hommes \& migrations}

Revue française de référence sur les dynamiques

migratoires

\section{1 | 2011}

Diasporas sri lankaises

\title{
Un champ de paradoxes
}

\section{Mustapha Harzoune}

\section{(2) OpenEdition \\ Journals}

\section{Édition électronique}

URL : http://journals.openedition.org/hommesmigrations/696

DOI : 10.4000/hommesmigrations.696

ISSN : 2262-3353

\section{Éditeur}

Musée national de l'histoire de l'immigration

\section{Édition imprimée}

Date de publication : 1 mai 2011

Pagination : 142-147

ISSN : 1142-852X

\section{Référence électronique}

Mustapha Harzoune, «Un champ de paradoxes », Hommes \& migrations [En ligne], 1291 | 2011, mis en ligne le 29 mai 2013, consulté le 22 septembre 2020. URL : http://journals.openedition.org/

hommesmigrations/696; DOI : https://doi.org/10.4000/hommesmigrations.696

Ce document a été généré automatiquement le 22 septembre 2020.

Tous droits réservés 


\title{
Un champ de paradoxes
}

\author{
Mustapha Harzoune
}

1 Les résultats du Front nationale (FN) aux cantonales et les sondages qui annoncent sa présence au second tour des présidentielles 2012 inquiètent. Marine Le Pen à l'Élysée, en lieu et place de la Pompadour... l'improbable avant-goût du mauvais goût. Pourtant, de nouveaux scénarios deviennent envisageables. Pourquoi pas une alliance avec de respectables "durs" de l'UMP par exemple? Patrick Buisson, ancien de Minute, un "des inspirateurs du thème de 'l'identité nationale' et du ministère du même nom" (Paris Match, 29 mars), et Maxime Tandonnet, "l'un des auteurs du discours de Grenoble", celui qui "a officialisé la notion de 'Français d'origine étrangère" - qui tient un blog aux idées jugées “ trop proches du FN" (Le Monde, 13 avril) -, conseillent l'Élysée.

2 L'immigration est dans le collimateur. Et le ton monte : déclarations à l'emporte-pièce, énième tour de vis législatif et Guéant qui donne le la. Marine Le Pen fanfaronne. Rien de plus facile. Pensée binaire et idéologie en mal de boucs émissaires évitent de discerner de quoi il retourne.

3 Comme dit Edgar Morin à propos de croissance : "Non! Il faut combiner croissance et décroissance." Et d'ajouter, toujours taoïste : "Je suis contre cette pensée binaire qui n'arrive pas à sortir d'une contradiction." L'immigration est un champ fertile en contradictions. Un champ aussi où l'arbre cache souvent la forêt. Les paradoxes entre les positions (de certains) politiques et les conclusions (de la plupart) des experts et études économiques ne surprennent qu'à moitié.

\section{Le Pen}

4 Alors donc Marine Le Pen y va de ses affirmations médiatico-télégéniques, autrement dit, comme ne cesse d'avertir Michel Rocard, spectaculaires, rapides, sans développements ni analyses, approximatives, voire mensongères. Ce qui compte, c'est la forme, pas le fond. Le 14 février, elle déclare: "Il y a un rapport du Conseil d'analyse économique (CAE) qui date de juin 2009 et qui dit que la hausse de $1 \%$ de l'immigration entraîne la baisse de $1,2 \%$ des salaires" (BFM TV). 
Ce rapport est celui de Gilles Saint-Paul, dont Michèle Tribalat fit grand cas dans son dernier livre. Comme d'autres, Mme Le Pen fait son marché. Elle choisit son boniment comme d'autres leurs salades. Quitte à travestir cette étude signée Altonji et Card qui montre qu'une hausse de l'offre de travail exerce une pression à la baisse sur les salaires des travailleurs de la même catégorie (quelle révélation !), en l'occurrence sur les bas salaires et non sur l'ensemble des salaires (tiens!), avec même un bénéfice pour les travailleurs complémentaires ( $a h ! a h$ !) et, dixit les auteurs de l'étude... une baisse du chômage (le pompon, non ?). Mais ce que ne dit pas non plus la fille de son papa, c'est que cette étude, qui date déjà de 1991, concerne la société américaine et pas la France et que les experts aiment à emberlificoter le citoyen et électeur en multipliant les "variables" retenues, oubliées, contestées, etc. Rigueur et complexité ne font pas bon ménage avec l'écran cathodique ni avec les tribunes des démagogues.

Le 13 mars, Le Républicain lorrain rapporte que, selon Marine Le Pen, janvier 2011 aurait enregistré une "extraordinaire poussée de l'immigration légale" par rapport à janvier 2009. “[...] Elle assure que le nombre de titres de séjour délivrés en janvier 2011 s'élève à 23 504, soit $42,1 \%$ de plus qu'en janvier 2010. Le nombre de demandeurs d'asile a également grimpé de 17,4\% (à 3 899) et celui des naturalisations de 19,4\% (à 13 337)." Et le quotidien de poursuivre sur les dénégations ministérielles. Le ministère conteste, apostrophe, morigène, mais c'est Marine qui est à la baguette.

7 Le 8 avril, Le Monde titrait sur une "hausse officielle des flux migratoires en 2009". "Ainsi, le nombre total de premiers titres de séjour a augmenté en 2009 de $3 \%$ par rapport à 2008, passant de 81600 à 82 700. La demande d'asile a augmenté de $11 \%$, soit 33200 demandes. Le nombre de visas étudiants a, quant à lui, crû de $3 \%$ avec un peu plus de 70900 titres délivrés. [...] L'immigration familiale [...] a connu une hausse de 1,4\%. Mais ce flux est essentiellement lié à l'entrée de conjoints de Français. Le 'regroupement familial' lui, qui vise les ressortissants étrangers, aurait par contre baissé de 16,8\% en 2009 et a concerné environ 13400 personnes. L'immigration professionnelle [...] a été victime, en 2009, du contrecoup de la crise économique" avec une baisse "de $6 \%$ en 2009". "Les visas pour établissement professionnel, au nombre de 16000 , ont baissé de près de $20 \%$."

8 Le 16 avril, le site Internet du Monde, citant cette fois "des statistiques de FTA et de l'Office français de l'immigration", annonce que "l'immigration légale en France a augmenté d'environ $10,6 \%$ en un an, à 188780 arrivants, sous l'effet notamment d'une forte hausse du nombre d'étudiants (65 840 personnes, en augmentation de 28,5\%)".

\section{Les dessous du vote FN}

9 Pour Laureline Dupont, dans Marianne ( $1^{\mathrm{er}}$ mars), Marine, comme papa, éructe "une rhétorique anti-immigrés façon années quatre-vingt". La preuve? Sa déclaration au salon de l'agriculture, le 25 février : "L'Union européenne est totalement impuissante à nous protéger, il faut passer un accord bilatéral avec l'Espagne et l'Italie pour permettre à nos marines de préserver nos eaux territoriales et repousser dans les eaux internationales les migrants qui voudraient entrer en Europe." Le $1^{\mathrm{er}}$ mars sur RTL, elle enfonce le clou: "On peut parfaitement repousser des bateaux dans les eaux internationales humainement."

10 Pour le sociologue Alain Mergier : "Le vote FN devient un vote positif d'adhésion à un point de vue, qui reconnaît [la] situation [de ces électeurs] et donne une valeur politique à ce qu'ils vivent." Et d'expliquer que "les classes populaires [...] n'arrivent plus à boucler leur budget 
mensuel. Elles ne vivent plus sous la menace, mais dans l'immédiateté, dans l'urgence d'une réalité qui devient invivable", alors que dans le même temps, elles observent "cette oligarchie financière qui aujourd'hui s'en sort très bien, ces entreprises du CAC 40 qui affichent des résultats historiques. [...] Cette dynamique-là, dans leur esprit, porte un nom: la mondialisation", et d'ajouter : "la question des flux migratoires constitue pour eux le second volet de cette mondialisation. Les nouveaux sympathisants du FN peuvent d'ailleurs avoir un discours très inquiet sur l'immigration, sans connotation raciste ou xénophobe : 'On n'arrête pas de nous dire qu'il n'y a plus d'argent, plus de boulot, comment peut-on accueillir tous ces pauvres gens ?' Une partie d'entre eux vivent dans les cités, les banlieues, où ils sont confrontés à des situations qui se sont dégradées" (La-croix.com, 27 mars).

11 Pour Emmanuel Todd, "les résultats [...] témoignent plutôt d'une prédominance des déterminations socio-économiques dans les votes. Le Front national a franchi son plafond ancien du vote à $30 \%$ dans la classe ouvrière et s'approche de $40 \%$ ". Selon le démographe, "deux signes confirment le caractère secondaire des thématiques identitaires (immigration, sécurité, etc.) : le score plus modeste (à $20 \%$, ce qui n'est quand même pas mal) réalisé parmi les artisans et commerçants et la progression importante du Front national vers l'ouest, là où l'immigration demeure un phénomène insignifiant". "L'ampleur générale du score frontiste et sa pénétration non insignifiante dans les classes 'moyennes inférieures' et 'moyennes moyennes', dans des cantons sans tradition industrielle et sans immigration, suggèrent l'idée que les gens n'ont plus confiance dans la gestion économique du pays" (Marianne2.fr, 24 mars).

D'ailleurs, à propos de la présidentielle de 2007, pour le politologue Stéphane Rozè, "ce n'est pas en parlant d'immigration, ou de sécurité, que Nicolas Sarkozy a gagné les voix du Front national. Mais plutôt grâce à la thématique du 'travail, du mérite et du pouvoir d'achat' [...]" (Le Monde.fr, 3 mars).

\section{Florilège}

13 Et pourtant, comme l'écrit L'Express.fr le 11 mars, "l'aile droite de l'UMP multiplie les discours musclés sur l'immigration ou la sécurité, défendant des thèses souvent proches de celles du Front national, au risque de semer la zizanie au sein de la majorité". Le 8 mars, la députée UMP Chantal Brunel déclare: "Il faut rassurer les Français sur toutes les migrations de populations qui viendraient de la Méditerranée." Et de lâcher : "Après tout, remettons-les dans les bateaux!" Claude Guéant, le nouveau ministre de l'Intérieur, fut le plus prolixe. Florilège de ses déclarations : "Les Français, à force d'immigration incontrôlée, ont parfois le sentiment de ne plus être chez eux [...]" (Europe 1, le 17 mars). "Les Français ont le sentiment qu'un excès d'immigration - une immigration irrégulière, non désirée - les trouble, les ennuit... [...] Ils ont aussi l'impression que cette immigration peut se traduire par des modes de vie qui s'imposent à eux et qu'ils ne souhaitent pas" (Talk-Orange-Le Figaro, 21 mars). "Les agents des services publics évidemment ne doivent pas porter de signes religieux, manifester une quelconque préférence religieuse, mais les usagers du service public ne [le] doivent pas non plus "(I.télé, 24 mars).

14 Enfin, dans Le Figaro Magazine du 8 avril, Claude Guéant annonce son intention de réduire l'immigration légale (regroupement familial et immigration de travail) et de commander "une étude' pour revoir éventuellement la procédure d'asile". Annonce confirmée et chiffrée sur le plateau de TF1. Objectif : diminuer de 20000 par an (sur 200000 ) le nombre d'étrangers autorisés à séjourner en France. Le ministre du Travail, Xavier Bertrand, annonçait le 17 avril au Grand Jury RTL-LCI-Le Figaro vouloir réduire la 
liste des métiers pour lesquels le recours à une main-d'œuvre étrangère est facilité. Pendant ce temps, après le débat sur l'identité nationale, Jean-François Copé remet le couvert avec un débat sur l'islam et la laïcité.

\section{"Assez ! Avec ça."}

15 Ces déclarations ne sont pas du goût de tous. Y compris dans les rangs de la majorité. Ainsi, Christine Lagarde, la ministre de l'Économie, déclare : "Je ne sais pas quelles sont les raisons qui ont amené [Claude Guéant] d̀ dire cette phrase [...] mais en ce qui concerne l'immigration qui est légale, évidemment il faut qu'elle soit protégée et sécurisée." Elle ajoute : "Dans le long terme, on aura besoin de main-d'œuvre, on aura besoin d'effectifs salariés formés."

Robert Badinter dans La Matinale de France Inter (7 mars) pousse un coup de gueule : “ Non! Pensons à ces millions qui sont nos compatriotes, qui sont musulmans, qui ont le droit de vivre comme les autres et qui n'aspirent qu'à cela. Eh bien oui ! Il y a une délinquance... Et alors? En quoi est-ce que ceci concerne ces millions, millions de citoyens français? Je rappelle ce que nous sommes, une République laïque, je rappelle ce que nous sommes, une République dans laquelle il ne doit y avoir aucune discrimination en fonction de la religion. Alors! Assez! J'ai entendu à ma stupéfaction parler de citoyens français, de compatriotes 'd'origine musulmane'. Vous imaginez ce que cela veut dire? Cela m'a ramené soixante ans en arrière, quand il y avait des Français ou des non-Français d'origine juive. Qu'est-ce que c'est que cela dans la République ? Assez! Avec ça."

17 Pierre Henry, directeur général de France terre d'asile, évoque "un climat de guerre civile ": "Le tissu social se déchire sous les mots et les faits produits au sommet de l'État. Ils disent la peur de l'autre, désignent des boucs émissaires et servent des intérêts électoraux plutôt que l'intérêt commun. Ils nous délivrent un récit national sur l'immigration qui fragmente notre société" (Le Nouvelobs.com, 11 mars).

Claire Rodier, directrice du Gisti (Groupe d'information et de soutien des immigrés), sur le site du Nouvelobs (8 mars) rappelle que la France est un État de droit et que "toute personne qui se présente aux frontières a donc droit à un examen de sa situation, même si par la suite il s'avère qu'elle ne peut pas prétendre à la protection internationale et qu'elle devrait éventuellement être renvoyée dans son pays en application de la loi nationale". D'ailleurs, "en 2009, [...] il y a eu des refoulements par bateau depuis l'Italie. Ce pays est maintenant poursuivi devant la Cour européenne des droits de l'homme. Le dossier vient d'être renvoyé devant la grande chambre de la Cour de Strasbourg".

19 Sophia Aram, humoriste chroniqueuse sur France Inter vilipendée pour avoir traité les électeurs du FN de "gros cons", se raidit: "Ces nouveaux apôtres, qu'ils s'appellent Philippe Bilger, Philippe Cohen, Robert Ménard, Elisabeth Lévy et j'en passe, nous imposent leur soudaine lucidité et nous enjoignent d'arrêter de diaboliser ces victimes qui se tournent vers un parti censé 'poser les bonnes questions, même s'il n'apporte pas toujours les bonnes réponses'. Le risque, c'est que, si vous prenez goût à poser les questions de la souffrance ou de la misère sociale à travers le prisme de la couleur de peau, de la culture ou la religion, alors vous allez adorer les solutions que sont le repli identitaire et la préférence nationale. [...] Quand l'origine et la couleur de peau deviennent les critères pour comprendre Le Monde, j'ai tendance à me raidir. C'est peutêtre parce que je suis viscéralement attachée aux valeurs républicaines et laïques, ce qui, compte tenu de mes origines, doit les surprendre" (Le Monde, 12 avril). 


\section{“Personne ne peut cacher le soleil avec un tamis ${ }^{1 "}$} nombre d'économistes. "Il faut plus d'immigrés, en France comme en Europe", lance Michel Godet, professeur au Conservatoire national des arts et métiers. "On a besoin de bras et de cerveaux et on s'enrichit de l'immigration." Selon lui, il va manquer "au moins 35 millions d'actifs d'ici à 2030" à l'Europe. C'est donc "une erreur de dire qu'il faut limiter l'immigration de travail, il faut au contraire la renforcer", car "un quart des emplois dans l'industrie ne trouvent pas de preneurs"(AFP, 9 avril). "Christian Saint-Étienne estime d'ailleurs que la France pourrait s'ouvrir davantage. 'Il faudrait que la France soit un aimant pour toutes les qualifications, dit-il. Si on veut s'inscrire dans une dynamique rapide de développement, on a intérêt à favoriser toutes les formes d'immigration de travail, un peu à l'américaine, comme le font les Australiens ou les Canadiens'" (AFP, 9 avril). 2050, [...] la France métropolitaine compterait 70 millions d'habitants, soit 9,3 millions de plus qu'en 2005. [...] En 2050, un habitant sur trois serait âgé de 60 ans ou plus, contre un sur cinq en 2005. La part des jeunes diminuerait, ainsi que celle des personnes d'âge actif. En 2050, 69 habitants seraient âgés de 60 ans ou plus pour 100 habitants de 20 à 59 ans, soit deux fois plus qu'en 2005. Ces résultats sont sensibles aux hypothèses retenues, mais aucun scénario ne remet en cause le vieillissement, qui est inéluctable."

Voilà pourquoi "les économistes Karine Berger et Valérie Rabault, (membres du PS)" diagnostiquent que "l'économie française a besoin de 10 millions d'immigrés d'ici à 2040" : " Selon nos calculs basés sur des projections de l'Insee, $26 \%$ des Français auront plus de 65 ans en 2040 si on ne modifie pas notre politique d'immigration', prévient Karine Berger". "L'idée est d'envisager [...] un flux de 300000 immigrés par an, soit 10 millions de personnes d'ici 2040 qui, selon nous, sont indispensables pour régénérer la population, permettre à notre économie de 
conserver sa capacité d'innovation et de pérenniser notre système de protection sociale" (La Tribune.fr, 21 mars).

\section{Renforcer l'intégration}

Face aux craintes de flux d'immigrés, Karine Berger et Valérie Rabault estiment "qu'un des moyens les plus efficaces pour intégrer ces immigrés est de 'mettre à bas la ghettoïsation'. Selon une étude réalisée par des chercheurs américains, le coût de la ghettoisation représente 3,8\% du PIB des États-Unis, indique Karine Berger. Rapporté au PIB français, toutes choses égales par ailleurs, on frôle les 80 milliards d'euros. On pourrait faire beaucoup de choses pour stopper ce phénomène"(La Tribune.fr, 21 mars).

Avec la crise et la plus grande vulnérabilité de certaines populations, il faudrait justement renforcer les politiques d'intégration. Et pourtant en Seine-Saint-Denis, “ Depuis le début de l'année, les vingt-huit écoles maternelles et primaires de la commune cumulent les absences non remplacées d'enseignants. [...] Une plainte a été déposée auprès de la Halde pour 'discrimination territoriale". "Une proposition de loi a déjà été déposée le 18 novembre 2010 par vingt députés communistes, du Front de gauche et divers gauche. Elle vise à ajouter le 'lieu d'habitation ou de résidence' parmi les motifs de discrimination" (Le Monde.fr, 13 mars).

De manière plus générale, "la Seine-Saint-Denis continue d'accuser un retard en matière d'éducation et de formation. À peine $10 \%$ des actifs disposent d'un niveau bac +2 , contre $40 \%$ à Paris ou $30 \%$ dans les Hauts-de-Seine. Près de $30 \%$ des actifs du département n'ont aucun diplôme, soit le double de Paris et des Hauts-de-Seine. Tous les indicateurs sont jugés préoccupants. Le nombre de jeunes sans le moindre diplôme est plus élevé. La proportion de diplômés de l'enseignement supérieur plus faible" (Le Monde.fr, $1^{\mathrm{er}}$ mars).

Et pendant ce temps, les religieux délivrent des leçons de laïcité républicaine. "Les représentants des principales religions pratiquées en France ont signé un texte dans lequel ils enjoignent au gouvernement de renoncer au débat sur la laïcité. Réunis au sein de la Conférence des responsables de culte en France [...] des représentants des bouddhistes, des catholiques, des juifs, des musulmans, des orthodoxes et des protestants demandent à l'exécutif de 'ne pas dilapider ce précieux acquis' qu'est la laïcité" (Le Monde.fr, 29 mars).

30 Ainsi prospèrent les paradoxes entre les besoins du pays et les déclarations ou les politiques mises en œuvre. Le temps long des migrations et des raisonnements se brise sur les exigences du court terme électoral et médiatique.

\section{NOTES}

1. "Ur yezmir yiwen ad yeffer itij s-uyerbal", proverbe kabyle. 